\title{
AdoBPRIM: Towards a New Healthcare Risk-aware Business Process Management Tool
}

\author{
Rafika Thabet $^{1,2}$, Amine Boufaied ${ }^{1}$, Elyes Lamine ${ }^{3}$, Dominik Bork ${ }^{4}{ }^{a}$, Ouajdi Korbaa ${ }^{1}{ }^{\mathrm{b}}$ \\ and Hervé Pingaud ${ }^{2}$ \\ ${ }^{1}$ University of Sousse, ISITCom, MARS Research Lab, Route G.P.1, 4011, Hammam Sousse, Tunisia \\ ${ }^{2}$ University of Toulouse, INP, CNRS, LGC, F-31432 Toulouse Cedex 04, France \\ ${ }^{3}$ University of Toulouse, IMT Mines Albi, Department of Industrial Engineering, Route de Teillet, 81013 Albi Cedex 9, France \\ ${ }^{4}$ University of Vienna, Faculty of Computer Science, Währinger Street 29, 1090 Vienna, Austria
}

Keywords: Medication Use Process, Medication Error, Risk-aware Business Process Management, BPRIM, ADOxx.

\begin{abstract}
Performing risk management in healthcare facilities is particularly difficult due to the highly dynamic, complex, and multi-disciplinary nature of healthcare processes like the Medication Use Process (MUP). Risk-aware Business Process Management (R-BPM) is a promising approach to obtain a better understanding of such processes by identifying and analyzing corresponding risks. However, not all R-BPM approaches perform well in capturing the complexity of clinical processes. In this work, we introduce a new R-BPM framework called BPRIM that allows the identification and the analysis of medication error risks related to the complex medication use process. BPRIM is implemented using the ADOxx meta-modelling platform and then tested in a real case study. The tool is specific to the case study, but the framework can be used also in other healthcare processes.
\end{abstract}

\section{INTRODUCTION}

Patient safety is a priority for several government agencies, non-profit organizations, and regulatory bodies considering the detrimental and financial consequences associated with Medication Errors (MEs) and Adverse Drug Events (ADEs). A ME is an unintended failure in the Medication Use Process (MUP) that leads to, or has the potential to lead to, ADE for the patient. Indeed, MUP is a complex and multidisciplinary process that involves numerous professionals and composes several steps. This complexity causes the occurrence of MEs, which can lead to serious health-related consequences for the patients. In 2015, the French National Authority for Health considered that $40 \%$ of the serious adverse events are of medication origin (HAS, 2013).

Many international studies and reports recognized that the majority of errors were not the result of reckless behavior on the part of healthcare providers, but occurred as a result of the speed and complexity of

a (i) https://orcid.org/0000-0001-8259-2297 the MUP. Reports concluded that many patients die during hospital admission as well as during primary care, simply due to medication errors (Moyen et al., 2008). Most of these errors are not coincidental but systematic in origin. Although most available studies originate from the USA, studies conducted in other countries such as the United Kingdom (Leape et al., 1991) and the Netherlands (Barker et al., 2002) have shown similar results. This strongly implies that medication errors are a major public health problem in many western as well as developing countries.

Reducing the MEs risk is a shared responsibility between patients, healthcare professionals, regulators, and the pharmaceutical industry at all levels of healthcare delivery. (Kane-Gill et al., 2017) recognizes that preventing harm resulting from adverse drug events may be circumvented by averting medication errors with appropriate risk management approaches.

What needs to change is the way in which healthcare risk management methods are orientated. Indeed, in the healthcare field, several risk management methods exist (HAS, 2013). However, these methods do not consider the complexity of MEs, its strong con- 


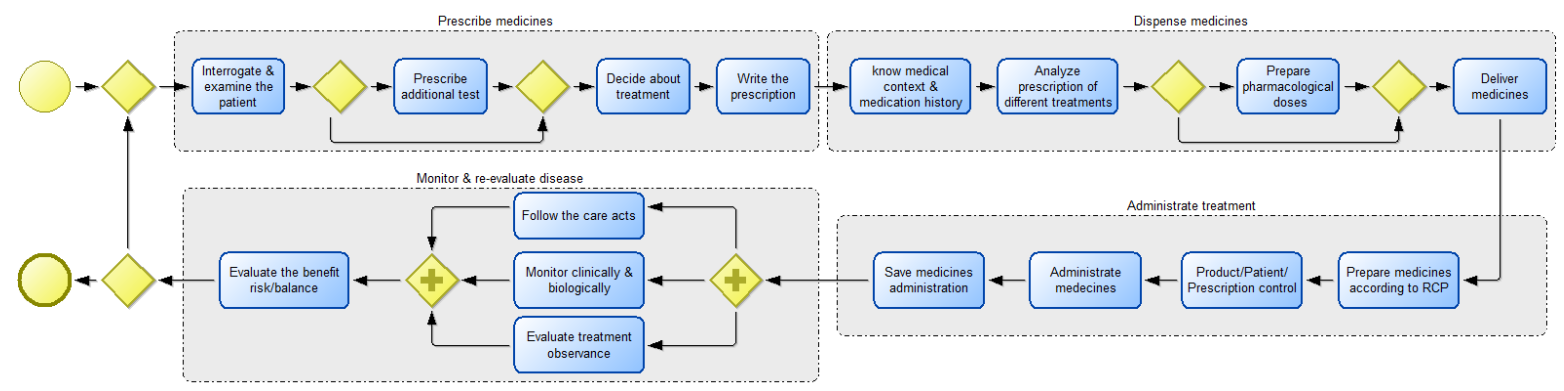

Figure 1: Medication Use Process as BPMN model (based on the French National Authority for Health report (HAS, 2013)).

nection to the MUP activities, and the influence of organizational and human factors. Following these observations, it would be essential for the healthcare facilities to explore new risk management approaches which consider all related concepts to the ME risk occurrence as well as their dependencies inherent to the steps and activities of the MUP.

To alleviate the shortcoming of existing approaches, we propose studying the potential of the Business Process-Risk Management - Integrated Method (BPRIM) (Lamine et al., 2019; Thabet et al., 2018) to manage MEs risks related to the complex MUP. BPRIM is an integrated management approach of risk and business processes following the Risk-aware Business Process Management (R-BPM) paradigm (Suriadi et al., 2014; Lamine et al., 2019; Thabet et al., 2018). R-BPM aims to integrate the traditionally separated domains of risk management and business process management. This integration allows increasing efficiency of the risk identification, detection, and assessment in relation to the business processes.

This paper is structured as follows. First, Section 2 provides an overview of the MUP and the impact and the frequency of the ME occurrence in this process. In Section 3, we propose a comparative study of the current risk management methods in healthcare. Our adopted framework and methodology are presented in Section 4. An overview of the first results obtained after the implementation of our framework is given in Section 5. Finally, Section 6 concludes the paper and outlines future perspectives.

\section{BACKGROUND}

In (Vest et al., 2019), the Medication Use Process is defined as "the fundamental system that provides the basis for safe medication use within the healthcare environment." Thus, ensuring medications are used and secured in the most appropriate manner and across all settings. The MUP consists of a complex and multidisciplinary process, involving numerous practitioners and several major steps (i.e., prescribing, dispensing, administration and medication monitoring).

Figure 1 represents the macro-steps and related activities of the MUP as defined in the French National Authority for Health report (HAS, 2013) by means of a Business Process Model and Notation (BPMN) model. Obviously, these activities do not reflect the reality. In reality, the MUP may involve up to 46 activities from the moment a doctor considers prescribing medication to the moment when this medication is actually administered or taken by the patient.

The complexity of this process increases the likelihood and number of occurrences of Medication Error (ME) risks. There is no consensus about the definition of a medication error. A systematic literature review found 26 different terminologies employed for a Medication Error (Lisby et al., 2010). As defined by the United States National Coordinating Council for Medication Error Reporting and Prevention (NCC MERP) (NCC MERP and Prevention, 2019), a medication error is: "any preventable event that may cause or lead to inappropriate medication use or patient harm while the medication is in the control of the health care professional, patient, or consumer. Such events may be related to professional practice, health care products, procedures, and systems, including prescribing, order communication, product labelling, packaging, and nomenclature, compounding, dispensing, distribution, administration, education, monitoring, and use". This definition is broad and suggests that errors are preventable at different levels.

These risks can be at the origin of Adverse Drug Event (ADE) with potentially severe clinical consequences for the patient. ADEs were defined as injuries resulting from medical interventions related to a drug. Adverse drug events may result from medication errors or from adverse drug reactions in which there was no error (Bates et al., 1995). ADEs consequences can be substantial, including hospital admission, prolonged hospital stay, additional resource utilization, and lower 
patient satisfaction (Caporossi et al., 2014). In this context, many publications have described the frequency and the cost of MEs and ADEs in the MUP (Bates, 2007). Starting in 2000, the Institute of Medicine began publishing a series of reports on quality in healthcare. The first one, "To Err is Human", increased the awareness of unacceptably high rates of MEs and sentinel events in U.S. hospitals with about 44,000 98,000 deaths annually attributed to these preventable mishaps (Committee on Quality of Health Care in America and Institute of Medicine, 2000). The second one, "Crossing the Quality Chasm", made four major points: errors are common and costly; systems cause errors; errors can be prevented and safety can be improved; and medication-related adverse events are the single leading cause of injury (Committee on Quality of Health Care in America and Institute of Medicine Staff, 2001). The most recent one, "Preventing Medication Errors", attempts to think about what needs to be done to reach the next level of medication safety (Committee on Quality of Health Care in America and Institute of Medicine, 2006). In this work, first, an epidemiological review showed that, estimated very conservatively, medications harm at least 1.5 million people per year. In hospitals, there are at least 400,000 preventable adverse drug events per year, or approximately one medication error per patient per day. That finding probably had the greatest impact on the general public (Committee on Quality of Health Care in America and Institute of Medicine, 2006).

The occurrence of these errors questions the robustness of the management methods actually used to secure the MUP in the healthcare facilities especially that knowledge and practices evolve and the organization must remain vigilant.

Facing the high frequency and impact of MEs and ADEs, healthcare facilities have to set up a risk management approach aiming to ensure patient safety, and specifically, to decrease ME occurrence related to the complex MUP. Section 3 provides an overview of some existing healthcare risk management methods.

\section{RISK MANAGEMENT IN HEALTHCARE FACILITIES}

In France, ten years ago, Experience Feedback Committees (EFCs), were created to analyze ADEs within a medical ward (Francois et al., 2013). An EFC is a multidisciplinary team representing the diversity of the functions encountered in the medical ward. The EFC members meet regularly to examine reported ADEs related to their medical ward. They prioritize ADEs either on the gravity of the event or on the frequency of occurrence and propose corrective actions. To analyze the ADE facts, the committee uses one or many of the existing risk management methods in healthcare facilities.

A comparative study of the most relevant risk assessment methods currently used in the healthcare domain is summarized in Table 1. This comparison is carried out in accordance with the following criteria:

- Process/Activity based: whether the method describes in which system process or activity the causes occurred;

- Reactive/Proactive: whether the method is reactive (risk considered ex post) or proactive (ex ante consideration of risk);

- Qualitative/Quantitative: whether the method follows qualitative and/or quantitative analysis;

- Input: the reasons for which the method has to start being applied;

- Causes Ranking: whether the method classifies different causes;

- Logical Relationships: whether the method describes relationships between multiple causes using logical operators to describe risk scenarios;

- Causes Chronology: whether the method provides a chronological reconstruction of causes;

- Causes Description: which description type is used to represent causes.

Generally, healthcare risk management methods can be categorized according to the used risk assessment type: Reactive risk assessment and Proactive risk assessment (see Table 1). Reactive risk assessment methods are Root Cause Analysis methods based on systematic questioning to identify the underlying causes of adverse occurrences.

According to Table 1, only few methods (three out of 10) tried to integrate system process or activity where the causes occurred in the analysis process. However, among these approaches, none provides a graphical description to represent system activities and relationships between multiple risk causes using logical operators. In fact, working with graphs allows to capture a large analysis scope.

In an organisation, all presented methods will set up brainstorming meetings with a team of healthcare providers, as well as quality/risk managers, to reduce the number of MEs and ADEs. However, from our point of view, none of these methods is able to satisfy all the following requirements:

- Deal with the complex MUP at the correct level by describing all related and collaborative activities that compose the process $(\mathrm{Rq} 1)$; 
Table 1: Comparing current healthcare risk assessment methods.

\begin{tabular}{|c|c|c|c|c|c|c|c|c|c|c|}
\hline Method & 总 & 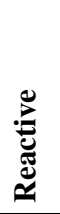 & 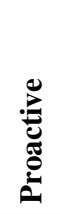 & 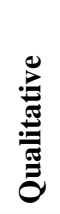 & 䒿 & $\overrightarrow{\mathbf{E}}$ & 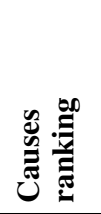 & 宽 & 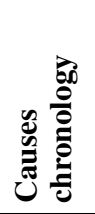 & 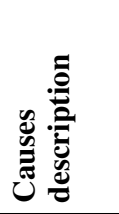 \\
\hline Five Whys? & $\square$ & $\square$ & $\square$ & $\square$ & $\square$ & Questioning & $\square$ & $\square$ & $\square$ & $\begin{array}{l}\text { Schema/ } \\
\text { Table }\end{array}$ \\
\hline Ishikawa & $\square$ & $\square$ & $\square$ & $\square$ & $\square$ & Questioning & $\square$ & $\square$ & $\square$ & Graph \\
\hline $\begin{array}{l}\text { Human Factor Analysis and } \\
\text { Classification System (HFACTS) }\end{array}$ & $\square$ & $\square$ & $\square$ & $\square$ & $\square$ & Questioning & $\square$ & $\square$ & $\square$ & $\begin{array}{l}\text { Graph/ } \\
\text { Grid }\end{array}$ \\
\hline $\begin{array}{l}\text { Association of Litigation And Risk } \\
\text { Management (ALARM) }\end{array}$ & $\square$ & $\square$ & $\square$ & $\square$ & $\square$ & Questioning & $\square$ & $\square$ & $\square$ & $\begin{array}{l}\text { Form/ } \\
\text { Report }\end{array}$ \\
\hline Causal Tree Analysis (CTA) & $\square$ & $\square$ & $\square$ & $\square$ & $\square$ & Questioning & $\square$ & $\square$ & $\square$ & Tree \\
\hline ORION & $\square$ & $\square$ & $\square$ & $\square$ & $\square$ & Questioning & $\square$ & $\square$ & $\square$ & $\begin{array}{l}\text { Table/ } \\
\text { Report }\end{array}$ \\
\hline $\begin{array}{l}\text { Failure, mode, effects, and criticality } \\
\text { analysis (FMECA) }\end{array}$ & $\square$ & $\square$ & $\square$ & $\square$ & $\square$ & $\begin{array}{l}\text { Identified } \\
\text { causes }\end{array}$ & $\square$ & $\square$ & $\square$ & Table \\
\hline Fault Tree Analysis (FTA) & $\square$ & $\square$ & $\square$ & $\square$ & $\square$ & $\begin{array}{l}\text { Identified } \\
\text { causes }\end{array}$ & $\square$ & $\square$ & $\not$ & Graph \\
\hline Event Tree Analysis (ETA) & $\square$ & $\square$ & $\square$ & $\square$ & $\square$ & $\begin{array}{l}\text { Identified } \\
\text { causes }\end{array}$ & $\square$ & $\square$ & $\square$ & Tree \\
\hline Preliminary Risk Analysis (PRA) & $\square$ & $\square$ & $\square$ & $\square$ & $\square$ & $\begin{array}{l}\text { Identified } \\
\text { causes }\end{array}$ & $\square$ & $\square$ & $\square$ & Table \\
\hline
\end{tabular}

- Identify and classify potential and reported ADEs and MEs (Rq2);

- Express ADEs in terms of combining potential factors, situations, events, and consequences (Rq3);

- Understand the strong relationships between the $\mathrm{ME}$ and activities of the MUP (Rq4);

- Consider the organizational and human factors which intervene the ME occurrence (Rq5);

- Produce a dynamic map ranking all ADEs (Rq6);

- Offer a well designed risk-process knowledge management repository $(\mathrm{Rq} 7)$.

- Provide the EFC team a modeling tool that also adheres to usability, performance, reliability, scalability, and availability requirements ( $\mathrm{Rq} 8)$.

Consequently, to advance the patient safety, healthcare facilities are required to implement an effective detection policy, prevention and control of MEs risks related to the complex MUP. To tackle this situation we suggest the exploration of new research domains, in particular the integrated management of risks and business processes.

\section{BPRIM FRAMEWORK}

Since several years a major research interest is given to integrate the two traditionally separated fields of risk management and business process management in a common concept known as R-BPM. The importance of this integration was confirmed by the research community (Zur Muehlen and Ho, 2005), in industry guidelines (COSO 2004), and in many literature reviews (Suriadi et al., 2014; Thabet et al., 2018; Lamine et al., 2019).

Business Process-Risk Management - Integrated Method (BPRIM) (Lamine et al., 2019; Thabet et al., 2018; Sienou, 2009) is one of the important approaches proposed in the R-BPM context. The method suggests an integrative approach with three components:

- A conceptual unification of risk and business process based on the coupling between the ISO/DIS 19440 conceptual model and a new conceptual model for risk.

- A BPRIM modeling language composed by abstract and concrete syntax. The abstract syntax is described by a meta-model extending the ISO/DIS 19440 constructs with a new set of constructs for 
(a) Risk Taxonomy Diagram

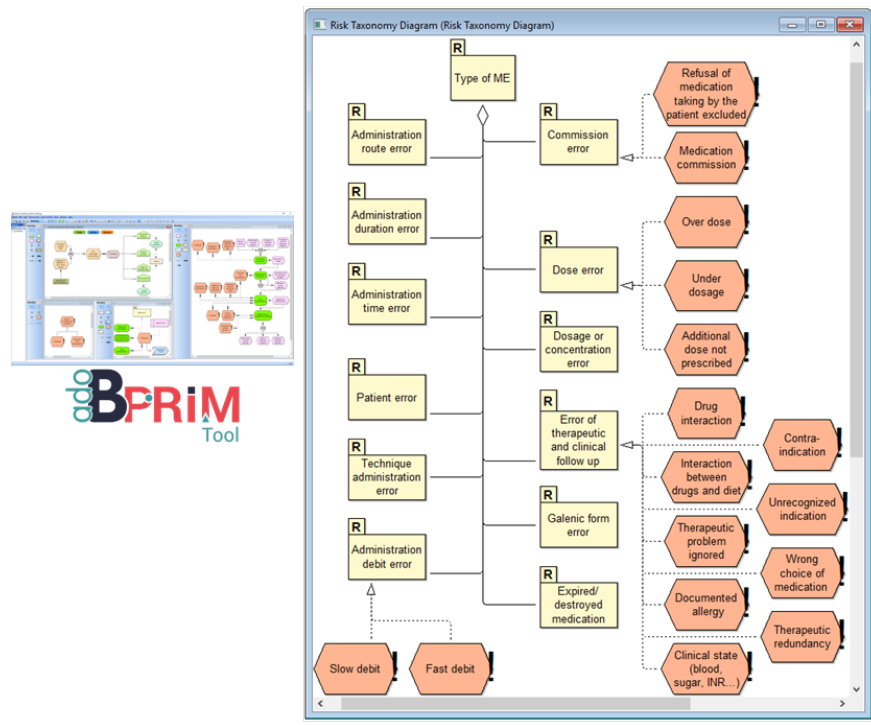

(d) Risks Map Diagram (b) Risk-extended EPC Diagram

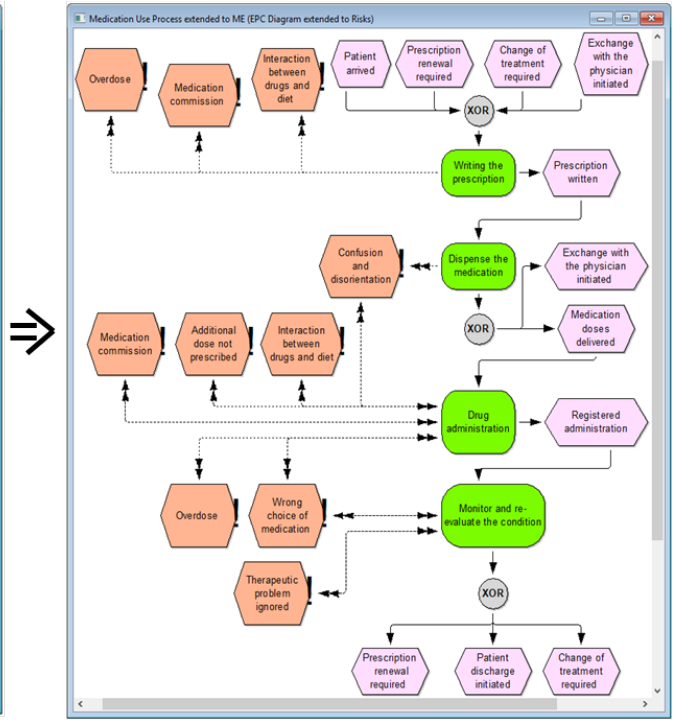

(c) Risk Analysis Diagram

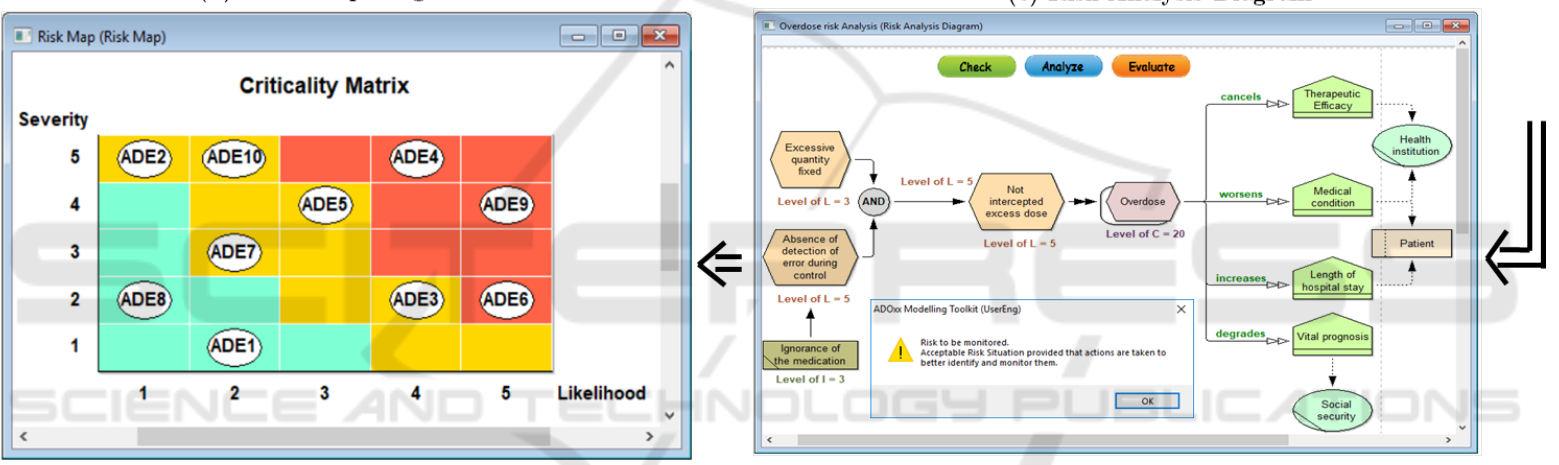

Figure 2: BPRIM Framework (excerpt)

risk modeling. The concrete syntax is proposed by extending the Event-driven Process Chains (EPC) notation. Figure 2 shows an extract of the BPRIM meta-model.

- A BPRIM lifecycle based on the coupling of the business process management and the risk management lifecycles. Considering information exchanged between the synchronized lifecycles of business process design and risk management, a set of diagrams was identified (e.g. Context, EPC, Risk-extended EPC, Risk Taxonomy, Risk, Risk Analysis, Risks Map) (see Figure 2).

In order to evaluate the potential of BPRIM and the ADOBPRIM tool, we specify for each part individually to what extent it satisfies the requirements defined in Section 3 .

\subsection{ADOBPRIM Tool Development}

The modeling tool supporting BPRIM called ADOBPRIM has been developed as a project within the Open Models Laboratory (OMiLAB), a worldwide community of modelers and modeling method developers (Bork et al., 2019). A free download and further information on ADOBPRIM are available through the corresponding project page ${ }^{1}$.

To realize the ADOBPRIM tool, respecting nonfunctional requirements $(\mathrm{Rq} 8)$ previously defined in Section 3, we have chosen the ADOxx meta-modelling platform (ADOxx.org, 2019). ADOxx has been widely used in industry and academia. In the past twenty years, more than 50 domain-specific modeling tools have been successfully realized with ADOxx (see (Karagiannis et al., 2016) for an overview).

\footnotetext{
${ }^{1}$ ADOBPRIM project page [online]: http://austria.omilab. org/psm/content/BPRIM/info, last visited: 16.12.2019
} 


\subsection{ADOBPRIM Models}

The main diagrams of our approach are outlined below and also in Figure 2 b, c, d, and e. Moreover, we highlight how each ADOBPRIM model contributes to the identified requirements:

Risk Taxonomy Diagram: This diagram aims to build an inventory of potential risks and to classify them according to various criteria. Risk identification can be performed using literature analysis and/or brainstorming with teamwork.

$\rightarrow$ Fulfilling requirement $\mathrm{Rq} 2$.

Risks-extended EPC Diagram: EPC diagrams originally describe business processes by means of related and collaborative activities that produce a specific service or product. Thereby, EPCs provide a comprehensive understanding of the system and enable process improvement. The Risks-extended EPC diagram aims to assign potential risks, defined in the Risk Taxonomy diagram to individual activities of the EPC diagram.

$\rightarrow$ Fulfilling requirements $\mathrm{Rq} 1, \mathrm{Rq} 4, \mathrm{Rq} 5$, and Rq7.

Risk Analysis Diagram: This diagram aims to analyze and evaluate individual risks. It includes: (i) Underlying causes and consequences; (ii) Risk level calculation, and (iii) Risk evaluation. Risk is a complex concept defined in different ways depending on the context and the discipline concerned. In our case, a risk $\mathrm{R}$ is expressed in terms of scenario S, likelihood L and severity $\mathrm{G}$ as expressed in Equation 1:

$$
R=(S, L, G)
$$

Risk scenario is expressed in terms of a combination of potential factors, situations, events, and consequences. Likelihood level is based on the expertise, knowledge and actual experience of the group scoring the likelihood. Severity of impact indicates the impact of the risk event to values interested for stakeholders. In this work, we are interested in qualitative risk analysis. A guide to likelihood and severity of impact levels is presented in Table 2. Definitions for the qualitative values were based on (BLONDEL and BRUNEL, 2004). The output risk level is illustrated in a two dimensional risk matrix. The purpose of risk evaluation is to prioritize the risks, and to decide which risks require treatment as well as the mode of treatment. $\rightarrow$ Fulfilling requirement $\mathrm{Rq} 3$.

Risks Map Diagram: This diagram is a two dimensional risk matrix showing the risk level of each analyzed risk.
Table 2: Likelihood and severity of impact levels according to (BLONDEL and BRUNEL, 2004).

\begin{tabular}{ll|ll}
\hline & Likelihood & \multicolumn{2}{|c}{ Severity } \\
\hline 1 & Very improbable & 1 & Minor \\
2 & Very unlikely & 2 & Significant \\
3 & Unlikely & 3 & Major \\
4 & Possible/Likely & 4 & Critical \\
5 & Very likely to certain & 5 & Catastrophic \\
\hline
\end{tabular}

$\rightarrow$ Fulfilling requirement Rq6.

Consequently, through models and capabilities of the ADOBPRIM tool of the BPRIM method, all functional and non-functional requirements defined in Section 3 are satisfied. Next, we will present preliminary results of the evaluation of the BPRIM framework and the ADOBPRIM tool in a real case study.

\section{PRELIMINARY EXPERIMENT RESULTS}

\subsection{Experiment Context}

To evaluate capabilities, feasibility, and relevance of the BPRIM framework and the ADOBPRIM tool, we use a real-world case study within an existing hospital. The study was conducted in the Pays d'Autan Hospital of the InterCommunal Hospital Center of Castres-Mazamet in France. With more than 388 beds, this hospital includes 30 wards.

According to the World Health Organization and (Kane-Gill et al., 2017), MEs and ADEs occur at a higher frequency and with a greater likelihood for elderly patients. Improving the quality of medication use and medication safety for elderly patients is thus an important public health priority. For this reason, our case study focuses on ADEs risks management related to the MUP for elderly patients in the Geriatric department of the Pays d'Autan Hospital. Our experimentation aims to: (i) check the feasibility of using the BPRIM framework to manage a serious of ADEs risks related to the MUP of elderly patients; (ii) experiment with the ADOBPRIM tool in a real case study; and (iii) identify potentials for improvement.

The experiment was carried out in collaboration with several actors of the Pays d'Autan Hospital, specifically two doctors, three nurses, two pharmacists, one pharmacy technician, and the Quality Manager. 


\subsection{Preliminary Results}

The repository of models developed during the experimentation currently holds around 40 BPRIM models. These last are created and validated in collaboration with the experimentation team.

The experimentation followed below steps:

1. Identify organizational units, roles and values related to the MUP and describe relationships between them in the context model. As a result, 8 organizational units, 10 roles and 6 values are presented in the context model;

2. Build AS-IS models of the MUP of elderly patients in the Geriatric department: First a valueadded chain model describing an overview of the MUP sub-processes is presented, and then each sub-process is defined by an EPC model. As a result, 14 EPC models presenting a detailed functional and organizational view of the MUP in the Geriatric department are presented. The EPC models involve up to 46 activities.

3. Identify potential and reported ADEs in the department. As a result, 16 ADEs organized in 12 classes are presented in the Risk Taxonomy model (see Figure 2 a);

4. Extend AS-IS models with potential and reported ADEs in the department. As a result, 14 EPC models extended with the 16 potential ADEs previously defined. Figure 2 b) shows an example of an Risk-extend EPC model;

5. For each ADE, analyze potential and reported facts, risk situations and risk events contributing to the ADE occurrence. We studied 10 serious ADEs risks, reported by professionals, related to the MUP of elderly patients and that had analyzed by the EFC using the ALARM method. As a result, 10 Risk Analysis models and 10 Risk models corresponding to reported ADEs are defined. Figure 2 c) shows the Overdose Risk Analysis model;

6. Evaluate each ADE using the risk matrix acceptance defined by (BLONDEL and BRUNEL, 2004) and produce a Risks Map model ranking all ADEs by risk level (see Figure $2 \mathrm{~d}$ );

7. Diagnose obtained results looking for potential improvements of the AS-IS models.

ADOBPRIM is a reactive/proactive integrated management tool of risk and business process management dedicated to facilitate and to support the multidisciplinary EFC members in their ADEs analysis exercise. Indeed, the tool supports them in understanding the MUP activities, identifying ADEs, understanding how the ADE has occurred, prioritizing remedial interventions, and possibly enhancing the MUP steps.

Moreover, with the ADOBPRIM tool, a database of potential ADEs, analyzes already carried out during previous EFCs can be accessed and augmented with new data. In addition, the tool allows accelerating the ADEs analysis process which consequently allows analysing more reported ADEs per EFC meeting. Indeed, due to the heaviness and the complexity of risk management methods currently used in healthcare facilities, the EFC committee chooses only one ADE per meeting in order to analyze it thoroughly.

The tool usage guidelines are partly defined by the BPRIM lifecycle. It should also be noted that a training session on the tool usage is necessary for a first use. Some models obtained by ADOBPRIM are presented like in figure 2. The latter effectively illustrates some of the models applied to the MUP extended to the risks of occurrence of MEs.

Validation and evaluation of our implementation results are currently performed in accordance with the very involved healthcare professionals of the Pays d'Autan Hospital.

\section{CONCLUSIONS}

The main contribution of this paper is the implementation of an effective approach to manage MEs related to the Medication Use Process based on the R-BPM concept. We presented an R-BPM framework for risk and business process management comprising the BPRIM method and the ADOBPRIM modeling tool.

The validity check for our contribution was performed in a real case study. The results motivate us to pursue the improvement of this method by integrating additional mechanisms and algorithms of risks analysis of the ADE related to the Medication Use Process of a patient within the healthcare facilities and studying the potential of the application of our framework in other healthcare processes as part of our future research.

\section{ACKNOWLEDGEMENTS}

The authors would like to thank the experimentation team from the Pays d'Autan Hospital of the Intercommunal Hospital Center (CHIC) of Castres-Mazamet and more specifically Dr. CUFI, the head of the Geriatric Department and Dr. CLEOSTRATE, the head of Inpatient Pharmacies who allowed us to evaluate our BPRIM method and ADOBPRIM tool on a real case and provided insights and expertise that greatly enhanced this research. We would also like to thank 
the OMiLAB team for assistance with the ADOxx platform and for providing a collaborative space on their OMiLAB portal.

\section{REFERENCES}

ADOxx.org (2019). The adoxx metamodeling platform. www.adoxx.org, last accessed: 17.12.2019.

Barker, K. N., Flynn, E. A., Pepper, G. A., Bates, D. W., and Mikeal, R. L. (2002). Medication errors observed in 36 health care facilities. Archives of internal medicine, 162(16):1897-1903.

Bates, D. W. (2007). Preventing medication errors: a summary. American Journal of Health-System Pharmacy, 64(14_Supplement_9):S3-S9.

Bates, D. W., Boyle, D. L., Vander Vliet, M. B., Schneider, J., and Leape, L. (1995). Relationship between medication errors and adverse drug events. Journal of general internal medicine, 10(4):199-205.

BLONDEL, P. and BRUNEL, C. (2004). Etude et hiérarchisation du risque infectieux nosocomial selon la criticité: nouvelle approche. Risques \& qualité en milieu de soins, (3):17-23.

Bork, D., Buchmann, R. A., Karagiannis, D., Lee, M., and Miron, E.-T. (2019). An Open Platform for Modeling Method Conceptualization: The OMiLAB Digital Ecosystem. Communications of the Association for Information Systems, 44:pp. 673-697.

Caporossi, A., Brudieu, E., Lehmann, A., Seigneurin, A., and François, P. (2014). An experience feedback committee for improving medication process safety: an observational study in a hospital pharmacy department. J Pharma Care Health Sys S1-010. doi, 10:2.

Committee on Quality of Health Care in America and Institute of Medicine (2000). To err is human: Building a safer health system. National Academies Press Washington, DC.

Committee on Quality of Health Care in America and Institute of Medicine (2006). Preventing medication errors: quality chasm series.

Committee on Quality of Health Care in America and Institute of Medicine Staff (2001). Crossing the quality chasm: A new health system for the 21st century. National Academies Press Washington, DC.

Francois, P., Sellier, E., Imburchia, F., and Mallaret, M. (2013). Experience feedback committee: a method for patient safety improvement. Revue d'epidemiologie et de sante publique, 61(2):155-161.

HAS, H. A. d. S. (2013). Outils de sécurisation et d'autoévaluation de l'administration des médicaments. SaintDenis La Plaine: HAS.

Kane-Gill, S. L., Dasta, J. F., Buckley, M. S., Devabhakthuni, S., Liu, M., Cohen, H., George, E. L., Pohlman, A. S., Agarwal, S., Henneman, E. A., et al. (2017). Clinical practice guideline: safe medication use in the icu. Critical care medicine, 45(9):e877-e915.
Karagiannis, D., Mayr, H. C., and Mylopoulos, J., editors (2016). Domain-Specific Conceptual Modeling, Concepts, Methods and Tools. Springer.

Lamine, E., Thabet, R., Seinou, A., Bork, D., Fontanili, F., and Pingaud, H. (2019). Bprim: An integrated framework for business process management and risk management. Computers in Industry, page in press.

Leape, L. L., Brennan, T. A., Laird, N., Lawthers, A. G., Localio, A. R., Barnes, B. A., Hebert, L., Newhouse, J. P., Weiler, P. C., and Hiatt, H. (1991). The nature of adverse events in hospitalized patients: results of the harvard medical practice study ii. New England journal of medicine, 324(6):377-384.

Lisby, M., Nielsen, L. P., Brock, B., and Mainz, J. (2010). How are medication errors defined? a systematic literature review of definitions and characteristics. International Journal for Quality in Health Care, 22(6):507518.

Moyen, E., Camiré, E., and Stelfox, H. T. (2008). Clinical review: medication errors in critical care. Critical Care, 12(2):208.

NCC MERP, N. C. C. f. M. E. R. and Prevention (2019). What is a medication error?

Sienou, A. (2009). Proposition d'un cadre méthodologique pour le management intégré des risques et des processus d'entreprise. $\mathrm{PhD}$ thesis, Institut National Polytechnique de Toulouse.

Suriadi, S., Weiß, B., Winkelmann, A., ter Hofstede, A. H. M., Adams, M., Conforti, R., Fidge, C., Rosa, M. L., Ouyang, C., Rosemann, M., Pika, A., and Wynn, M. (2014). Current Research in Risk-Aware Business Process Management - Overview, Comparison and Gap Analysis. Communications of the AIS (CAIS), 34(January 2014).

Thabet, R., Lamine, E., Boufaied, A., Korbaa, O., and Pingaud, H. (2018). Towards a risk-aware business process modelling tool using the adoxx platform. In International Conference on Advanced Information Systems Engineering, pages 235-248. Springer.

Vest, T. A., Gazda, N. P., Schenkat, D. H., and Eckel, S. F. (2019). Practice-enhancing publications about the medication use process in 2017. American Journal of Health-System Pharmacy.

Zur Muehlen, M. and Ho, D. T.-Y. (2005). Risk management in the bpm lifecycle. In International Conference on Business Process Management, pages 454466. Springer. 